\title{
A Method in Collaborative Hybrid Routing Protocol for Next Generation WSN by using Fuzzy Logic
}

\author{
Ram Bhushan Agnihotri, Nitin Pandey, Shekhar Verma
}

\begin{abstract}
Remote sensor systems will be frameworks of free halfway fact points castoff for inspecting a state of affairs. Specialists of WSNs face troubles that rise up out of correspondence interface disillusionments, memory and computational goals, and confined imperativeness. Various issues in WSNs are point by point as multidimensional streamlining issues, and moved closer through bio pushed strategies. There are various strategies which are fundamental, fruitful, and computationally capable improvement estimation. It has been associated with location WSN issues, for instance, perfect association, center constrainment, Clustering, and statistics accumulation. Thusly, this proposition work plots problems in WSNs, well-knownshows usually energized figuring which assist us hip improving the freestyle lifespan of the Feeler middle points, and appears at its fittingness for WSN bids. It furthermore affords a short audit way the usually impelled estimations are uniquely suited to deal with these issues. Bio breathed lifestyles into upgrade strategies are computationally succesful picks in preference to logical systems.

Sensors are seen as imperative pieces of electronic contraptions. In much use of remote sensor frameworks (WSNs), imperative and essential information must be passed on to the sink in a multi-bob and essentialness successful way. See that the essentialness of sensor center points is limited; hauling out framework lifetime in WSNs is seen as a fundamental issue. In order to extend the framework lifetime, authorities should consider imperativeness use in directing shows of WSNs. In this paper, another essentialness capable guiding show (EERP) has been proposed for WSNs using A-star figuring. The proposed coordinating arrangement improves the framework lifetime by sending data distributes the perfect most restricted way. The perfect way can be found as for the most extraordinary outstanding imperativeness of the accompanying hop sensor center, high association quality, support inhabitance and least skip counts. Amusement results show that the proposed arrangement improves compose lifetime in connection with A-star and feathery logic $(A \& F)$ show.
\end{abstract}

Keywords: Wireless Network, Fuzzy Routing Protocols, Energy efficiency, routing optimization.

\section{INTRODUCTION}

\section{Background Of The Study}

Web of Things (IoT)are dynamically being grasped into practical packages, as an instance, security structures, sharp establishment, site visitors the officers, ecosystem systems, amongst others. While the quantity of those packages is significant, tool limits, in particular in regards to battery lifestyles and imperativeness efficiency are confined. Regardless of research being completed to enhance those shortcomings, faraway IoT kinds out nonetheless can not guarantee desirable framework lifetimes and deferred recognizing consideration. Moreover, proposed contrives recorded as a hard reproduction are tangled and cannot be appropriately completed in certified situations. This requires the development of a clear but essentialness feasible coordinating association for far flung IoT feeler frameworks. This tabloid copies the essentialness restriction issue of diplomacies in IoT bids as an improvement issue. Near direct imperativeness of devices, the anticipated show uses gathering, pack head choice and least essentialness expensive route count for powerful and continuous guiding.

\section{SCOPE OF WORK}

Remote sensor hubs are typically fueled by batteries and passed on in unmanned outside or risky districts. Thusly, obliged essentialness is a recognizable part for remote sensor frameworks. Since the radio handset usually eats up a more prominent number of energies than some other gear fragment on a sensor center, it is of staggering hugeness to structure essentialness streamlined directing figuring to postpone orchestrate lifetime. In this work, in light of examination of imperativeness use for data handset, single-bounce sending plan is shown to eat up less essentialness than multihop sending plan inside the correspondence extent of the source sensor or a current forwarder, using free space imperativeness usage show. We grasp the social welfare ability to predict uniqueness of waiting imperativeness of neighbors in the wake of picking different next bounce centers. In light of imperativeness lopsidedness, the method is planned to enroll the dimension of essentialness balance. Parameters, for instance, dimension of closeness of center point to the most restricted way, dimension of closeness of center point to Sink, and dimension of essentialness balance are put into soft reason structure. Fleecy method of reasoning based imperativeness updated coordinating count is proposed to achieve multi parameter, soft guiding decision. Propagation results show that the estimation satisfactorily widens the framework lifetime and has achieved essentialness profitability and imperativeness balance together, differentiated and equivalent figurings. 


\section{A. PROBLEM STATEMENT}

Productive administration of essentialness sources is a tough studies locale in Wireless Sensor Networks (WSNs). Late examinations partake found out that grouping be situated a powerful topology manipulate technique for managing a framework right into a related motion which changes the site visitors save of the feeler centers and rallies the inclusive flexibility and the epoch of WSNs. Impelled through the upsides of bundling strategies; we have three essential obligations in this broadside. In the primary location, we recommend an essentialness feasible collecting development estimation called Active Node Cluster Formation (ANCF). The center imply on the manner recommend ANCF figuring is to fitting overpowering statistics flow and excessive essentialness utilization load similarly within the framework by means of subscription inadequate period of clusters inside the framework. The made association chooses every $\%$ head $(\mathrm{CH})$ close to the sink and distinguishing occasion even as the remaining affiliation of the gathering heads $(\mathrm{CHs})$ are appointed in the midst of each bundle deal to gain the maximum uncommon measure of essentialness viability in thick affiliation. Second, we propose a mild-weight identifying tool called Active Node Sensing Algorithm (ANSA). The key intend to recommend the ANSA figuring is to hold up a key separation from excessive identifying covering information redundancy with the resource of assigning a ton of dynamic middle factors in every accumulating with satisfy incorporation close to the occasion. Third, we suggest an Active Node Routing Algorithm (ANRA) to cope with complex bury and intra percent directing troubles in appreciably thick sending reliant on the middle aspect administering traits. Expansive exploratory examinations drove via framework check framework NCTUNs 6.Zero reveal that our proposed arrangement outmaneuvers current coordinating systems to the extent imperativeness profitability, from starting to prevent deferment and information redundancy, impede the directors and setup manipulate.

\section{INTRODUCTION}

Cutting-edge faraway feeler organize, we entail of minimum dimensions feeler policies that can use confined free style manipulate. Precisely when Wireless beam mastermind is sent in spotting locale at that point it accumulates really weird information and send to big middle factor referred to as sink. [1] The sensors in a Wireless sensor set up aren't gifted to be invigorated when their battery channel out of pressure which reasons a couple of problems, for example, correspondence opening, and so on so this is the purpose we use strategies to be used like to use compact sensors to trade their location from excessive imperativeness stage to low essentialness stage. [2] A bendy sink is relocated its scenario as opposed to sensor middle factor. In Wireless sensor mastermind, we've got controlling display inert and energetic. In inert guiding display the feeler center leads the statistics thru predefined footpath to the sink.[3] but in unique display the way can be demarcated for diffused the records to present day kingdom of the beam center, as such it is able to alter the stack on every feeler middle, we use thoroughgoing incredible cutoff way, which increase the framework epoch.[4]

In remote beam orchestrate, when immobile Wireless sensor kind out is despatched in an identifying an extent, each sensor middle has steady position and distinguishing the facts pass by manner of hop in view of which sensor middle channel out their battery essentialness. [5] except in relocate Wireless radar orchestrate, the bendy sensor can pass their territory whilst beam center aspect exhausts out their whole scale imperativeness. Throughout the most today's multi yr, we use diverse strategies. In Energy Aware Sink Relocation, we discover the transmission volume of every beam node.[6] According to their more battery essentialness it includes transmission continue to sink motion. Atom Swarm Optimization is a essential, fruitful and computationally worthwhile improvement algorithm.[7] In Constituent part Horde Optimization figuring, a swarm of atom starts offevolved to fly in chase location, by way of then we decide the execution of every atom as demonstrated through their aim work. Particle having quality revel in secured in a reminiscence called singular quality. The person excellent set the estimation of typical excellent.[8]

\section{Wireless Sensor Network}

Remote Sensor Systems are built up with little electro mechanical devices for instance sensors center points. Sensor center points give through RF signals with no less than one pivotal sinks called base stations (BSs). [9] Communication can be called single desire if they can contact direct to base station; or can be Multihop correspondence in which transitional center points shares for correspondence from sensor center point to assemble station.[10] Formally sensor frameworks concerning reason of their value are orchestrated into two sorts one is proactive and the other is responsive frameworks. Proactive frameworks are latent in nature and suitable for data complete sort applications.[11] In this kind of frameworks center points sense and send data on periodical reason i.e.[12] on standard between times. In Reactive frameworks, converse to inert frameworks sensor center points respond instantly and just to changes in the relevant parameters of intrigue.[13] Responsive frameworks are dynamically suitable for time fundamental applications. To haul out lifetime of sensor frameworks it will be progressively beneficial if sensor center points can collaborate with one another.[14]

That is the inspiration driving why sensor frameworks need the load up planes. As per need three organization planes will work which are named as 2 control organization plane, compactness the administrators plane, and errand the officials plane. [15] These planes grasp targets like; first to make sensor center points work all things considered in a power viable way, second to perform coordinating of data in remote sensor mastermind, and to share resources among them.[16] Without these three planes, each sensor center will act and capacity as an individual. Power the administrators plane certifications to regulate in what limit should a sensor center point uses its ability like when to murder beneficiary; when to convey low power message and to prevent from directing so sensor center points can save imperativeness for recognizing. [17] The adaptability the officials plane is being utilized when sensor center points are not stationary in frameworks. It perceives and selects the improvement of sensor center points. Adaptability plane keeps up a course back to the customer, and moreover sensor centers could screen their neighbor sensors.[18]

The endeavor the load up plane parcels and timetables the assignments among sensors 
which are spread in checking region. It isn't continually required that all sensor center points keep distinguishing in the meantime in a specific territory. It is similarly possible that some sensor center points play out the commission further than the others reliant upon their ability side by side.[19]

\section{LITERATURE REVIEW}

[20] In this paper, we have structured three vitality advanced parameters, for example, the level of closeness of hub to the briefest way, level of closeness of hub to Sink, and level of vitality equalization, and put these parameters into fluffy rationale framework. fluffy rationale based directing calculation is proposed to acknowledge vitality advanced, multi parameter, and fluffy steering choice. [21] Average vitality utilization of start to finish. Calculation expands the system lifetime viably contrasted and comparable calculations for various dada age designs and has a decent act regarding vitality equalization and vitality effectiveness. Our future work will concentrate on the applications for mufti media.[22] While accomplishing advanced vitality utilization of the entire system, the Qos, for example, transfer speed, inactivity, and bundle misfortune rate will be considered to meet the prerequisites of explicit applications.

[23]In a far flung sensor organize (WSN), the place across the Sink frames a bottleneck area wherein the site visitors circulation is maximum excessive. In this way, the epoch of the WSN arrange remains directed via the epoch of the tailback area. Circulating the power load a number of the hubs builds the epoch and nature of the system. Our recreations demonstrate that PEGASIS plays superior to $\mathrm{LEACH}$ via round one hundred to three hundred $\%$ whilst $1 \%, 20 \%$, half of, and a hundred $\%$ of hubs chew the dust for various device sizes and topologies. PEGASIS demonstrates a considerably further development because the degree of the gadget will increase.[24] In request to check our suspicions about PEGASIS, we are able to enlarge the gadget check machine ns-2 to mimic PEGASIS, LEACH, and thru spread conventions. In light of our $\mathrm{C}$ reenactments, we count on that PEGASIS will beat the other two conventions as a long way as framework lifetime and the nature of the community.[25] [26] In tabloid, a Minimum Energy (MINEN) steerage conference for IoT-WSNs is projected. MINEN be situated a bunching typically constructed eleven calculation which uniformly disperses the fee of energy utilization among each one of the gadgets of the machine. This is finished the use of bunching, institution head pivot and minimization of the strength of sending and getting messages crosswise over connections sincerely as helping diplomacies with short lingering verves.[27] This is executed through first growing a DAG in which the hubs of the chart are the group pâtés. At that factor becoming charges/hundreds are allocated to the edges remembering the power required for transmitting/getting messages over a specific connection in reality as a aspect for a tool pair framing a correspondence interface, termed Energy spent up till now (Esf). Incorporation of the Esf component upholds power based totally burden adjusting over a few connections within the software. [28]We had displayed guides to indicate working of MEDC convention. Recreation for MEDC is done on MATLAB. For assessment of this grouping convention organize lifetime criteria is taken, Results of MEDC are contrasted and HEED; and it is demonstrated that MEDC is performing superior to HEED. MEDC convention has appeared in system life time.[30] We had additionally appeared of MEDC under different parameters.[29] Forth section is about our second contributory work named MEHEED. MEHEED convention is combined arrangement of two grouping convention one is MEDC and the other is HEED. [30] This part will initially display idea of MEHEED bunching convention then calculation after that test results under different parameter. Execution of MEHEED is contrasted and MEDC and HEED as well.[31]

\section{A. EXISTING SYSTEM}

B. As of late, a extensive range of conventions had been proposed for far off structures that beautify safety problems, improve arrange unwavering quality and lift power productivity. AI and diversion hypothesis have likewise been used in coping with coordinating responses for far flung frameworks. As a end result of the essentialness [32] considerations associated with maximum battery primarily based far flung frameworks, hauling out framework lifetime is a key difficulty. This has meant an extended studies excitement for confirmation of a green faraway IoT network.[33] The makers communicate about the need for inexperienced IoT and the special programming and equipment based totally headways required to have interaction its affirmation.

C.Imperativeness succesful among center point correspondence and improved coordinating frameworks have been recognized because the problems that ought to be advised to empower extensive scale appointment of green IoT [34] There exist multiple coordinating indicates and framework sending plans which have been deliberate for WSNs. In guiding suggests for WSNs had been organized into 3 exciting social activities: Flat coordinating, region based controlling and one-of-a-kind leveled coordinating The paper displays an expansive research of coordinating three shows arranged beneath all of those 3 get-togethers. Level controlling shows are statistics pushed, which surmises that there are not any stringent tips on the lowest of the information. [35] Sensor middle factors in such shows cooperate to carry out identifying errands and statistics is addressed by means of manner of the usage of the lowest station from one of a type land regions Location based totally steering conventions like vicinity sensor hubs by way of their regions. This is made achievable via one in each of a type techniques at the side of relative separation estimation the use of sign traits or facts change and GPS based completely vicinity following. [36] The last elegance of conventions is significant to the trouble of energy protection. Various leveled steerage is the class of conventions which effectively partition device's directing duties based totally on machine capacities. High vitality hubs are relegated with the errand of records getting ready and statistics alternate, at the same time as low vitality devices are entrusted with detecting the earth. [37] Cluster primarily based totally steering techniques are likewise assembled underneath various leveled directing due to the fact the unique data making ready and transmission errands are appointed to the selected corporation heads. The proposed directing element on this paper businesses gadgets primarily based on severa large highlights, as an 
example, the separation of the hub from the lowest station, lingering battery dimensions of the devices, period of messages created and diploma of information detected with the useful resource of the sensor nodes.[38]

\section{PROPOSED SYSTEM}

The progression of the proposed least vitality (MINEN) steering convention is portrayed in Figure 1

1. The significant strides of the convention can be outlined as pursues:

- Running rest making plans to distinguish hubs now not taking aspect in the gift age. This development is discretionary due to the reality the execution of the convention is also tested without using rest scheduling.[39]

- Cluster improvement and bunch head race

- Edifice of DAG interfacing altogether bunch heads \& figuring of detail hundreds

- Seriatim Dijkstra to differentiate bottom price manner to lowest station for the present age For presumptions made thru the convention are:

- All devices start with a similar length of power

- There is excellent a unmarried BS located at a stationary characteristic in IoT set up

- BS is idea to be provided with in no manner-ending diploma of electricity, as an example a base station isn't always at the threat of final down due to absence of vitality [40]

- A series of correspondence is belief to be the timeframe among race of new organisation heads in the device and fruitful program of communications from all bunch pâtés to the lowest post.[41]

Rest Scheduling

Rest making plans is carried out to differentiate inactive devices which want to be controlled off within the path of the start of every correspondence spherical, to spare energy. As referenced previously, on this paper we use GSO for relaxation planning, that is a aggregate of PSO and GA. [42] These transformative enhancement methods classically improve thru the accompanying grouping of steps:

1)

Create an underlying population of the system randomly

2) Calculate the respect for one good for every association. It is well respected that the closeness of Vijay's answer to the right system should be depicted

3) Reconstruction and adjustment of various types of population in preparation based solely on their well-being esteems

4) Repeating the tool from degree 2 till conditions for the first-class association were met A everyday affiliation is taken into consideration as a Boolean exhibit of the span of the quantity of hubs in the network.[43]

Individually file of the show off includes a Boolean esteem connoting whether or not the machine at that file have to be located to rest (real) or not (fake) A lot of such clusters is alluded to as a population of arrangements. This populace is haphazardly brought initially and is changed utilising a whole lot of directors till the very last association is completed. The evaluation amongst PSO and GA lies in the way this inhabitants of arrangements is reformed.[44]

\section{METHODOLOGY}

A Fusion Dynamism Cognizant Course-plotting Set of rules for Wireless Sensor Networks
To expand the lifetime of the sensor networkanother coordinating method which solidifies two figurings: LEACH and PEGASIS In our advice, center points having a spot with a comparative cluster shape a chain where center factors communicate about simply with their closest associates, so imperativeness dispersal inner gatherings may be constrained and subsequently, life of the framework Can be better-quality. Reenactments using Network Simulator ns2 were directed to consider the lead of our coordinating calculation.[45] Sensor Network Hybrid Routing Protocol for Prolonged Network Lifetime in Large Scale Wireless theChain based virtually LEACH display in sensor frameworks have forced essentialness and in first rate scale sensor frameworks, correspondence between sensor center factors is critical to cover a generous looking district, thusly imperativeness sparing techniques are smooth. A waft breed show, which we are able to name all topics taken into consideration Chain-based totally honestly truely LEACH that improves the Low-Energy Adaptive Clustering Hierarchy to out and out lessen essentialness utilization and addition the life of a sensor put together. Our show uses LEACH and the upsides of Power Efficient Gathering in Sensor Information Systems and avoids their terrible elements.[46] LEACH technique improves imperativeness functionality of a sensor compose with the resource of choosing a clusterhead, and having it all out information from diverse middle points in its accumulating, and PEGASIS is a close-by first-class chain-based totally absolutely display that we used for correspondence and in addition aggregation amongst percentage heads which can be buddies and substitutes transmitting to the sink. Reenactment consequences show that CBL performs higher than LEACH.[47].

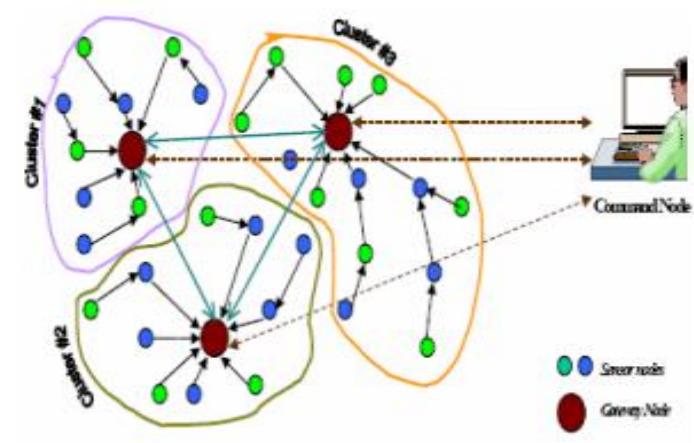

Figure 1 LEACH

Power-Efficient Gathering in Sensor Information System Get-collectively recognized facts in an imperativeness succesful manner is crucial to paintings the sensor put together a noteworthy part of time. In information accumulating problem is portrayed wherein in a improvement of correspondence, every sensor center detail has a package deal deal to deliver to the remote base station. If each center transmits its identified information simply to the bottom station, via then it's going to burn up its potential speedy. The Leach display displayed in a lovely path of motion in which clusters are encircled to consolidate facts earlier than transmitting to the lowest station [48] By randomizing the collection is going to transmit to the bottom station, Leach achieves a trouble of eight development appeared in each other manner close to coordinate transmissions, as assessed the amount that once middle factors kick the can. [49] Power-Efficient Gathering in 
sensor information systems, a nearby extraordinary chain-primarily based display this is an improvement over LEACH. In PEGASIS, every middle examines simply with a close-by neighbor and exchanges transmitting to the lowest station, alongside these strains diminishing the percentage of imperativeness spent in step with round.

Distributed Data Gathering Scheduling in Multi-hop Wireless Sensor Networks for Improved Lifetime

It unconstrained chooses a records amassing plan from the sensors toward the lowest station the idea. No common finding out about the topology is wanted for the trouble and except every sensor does no longer want to get to the bottom station at once.[50] Simulation contemplates display off that with admire to form lifetime, the proposed figuring internal and out outmaneuvers the arranging venture to the Minimum Spanning Tree (MST), or the Shortest Path (SP) guiding strategies. Execution examination with PEGASIS indicates that the proposed computation performs higher as a commonly developing form of middle factors save you to exist.

\section{$[57,58]$}

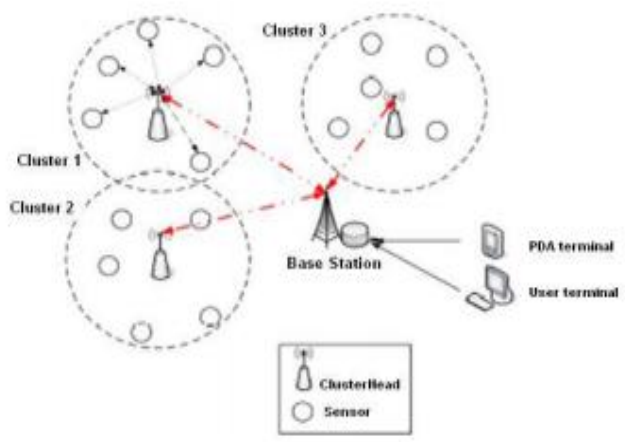

Figure 2 Clustered wireless sensor network

Lifetime Enhancement in Wireless Sensor Networks through Selective Data Handover

Subsequently, sensible indicates need to be portrayed to constrain the essentialness scattered thru the character middle factors in the framework. The Leach and PEGASIS initiation indicates are super responses for the hassle. While the Leach show randomizes bunch devices out in the path of identical essentialness dispersal, the PEGASIS show outlines a sequence of package deal deal heads taking rounds in transmitting to the lowest station.[51] The twofold dynamic version furthermore addresses this hassle. We endorse Data Handover Scheme which improves the execution of LEACH, PEGASIS and twofold version just like framework lifetime. The base station being organized at variable divisions from the individual middle factors, paying little mind to randomization and chain challenge plan, each center element really scatters a alternative percentage of imperativeness in the midst of its turn of transmission to the lowest station. This imperativeness differentiate will growth due to the reality the lowest station is decided in addition off from the recognized location and straightly with the amount of cycles snuck beyond and the \% period. [52] DHS clears out this imperativeness differentiate with the useful useful resource of facts handover in unequivocal pushes thru appropriate center trouble coordinating and assistant swapping.

Coverage Lifetime Improvement in Wireless Sensor Networks by Novel Deployment Technique

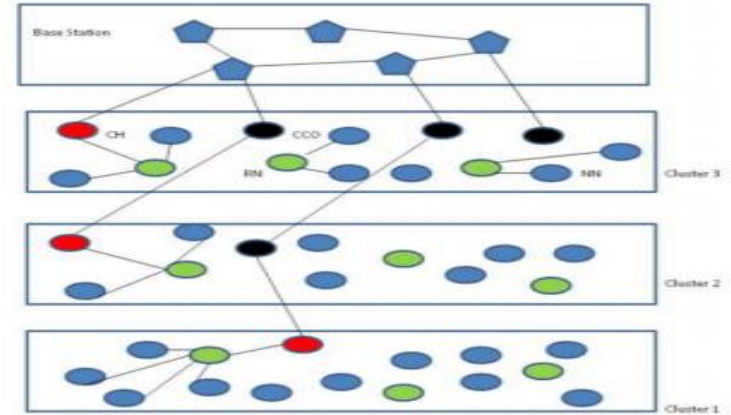

Figure 3 Multihop Wireless sensor network

[Fifty three] The quantity of sensor center points has direct impact at the price of the Wireless Sensor Networks. Thusly, recollecting this, the right course of movement of middle points can reduce the multifaceted concept of problems, imperativeness utilization may be diminished and in the long run widens the existence of the Wireless Sensor Networks. We are thinking about the important component situation, so the sport plan of sensor center breezes up sporadic. We are molding the enhancement of the sending as Rhombus apart from Triangle, Square and Hexagon which may be taken preferably for passing on the sensor middle elements and within the wake of the use of the proposed technique the existence of the given framework will growth.[54]

\section{RESULTS}

We mimicked MINEN, LEACH and FCM for relative research based mostly on :

- Number of alive hubs: This appraisal watches the diploma of viably taking part middle elements thru the improvement of correspondence rounds. The higher the amount of dynamic units, the more can be the efficient furthest reaches of the framework.

- Energy elements: A power rather than extensive fashion of rounds (time) correlation has been finished to examine the quantity of rounds for which the strength of a machine keeps going. [55]

- Network Coverage: This is used to examine the land incorporation of the going for walks devices of the framework thru the improvement of time. It will in stylish be defined as the quantity of rounds for which devices are viably including to the IOT framework's motion in a particular geological district.[56]

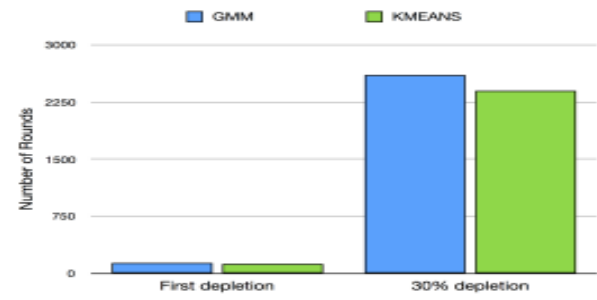

Figure 4 Clustering algorithms comparison

Table demonstrates the technology parameter's instatement regards which have stayed utilized in examination of the effects of the proposed show on this fragment. We have inspected the execution of MINEN with and without the use of relaxation arranging. It can be visible that even without the greater imperativeness guarantee manual of relaxation arranging, MINEN beats LEACH and FCM with the useful useful resource of crucial 
edges. Since MINEN, LEACH and FCM perform randomized batching, particular consequences can be gotten every time the estimations are run. Subsequently, the effects confirmed up on this section are the outstanding outcomes gotten for MINEN. Results for FCM and LEACH are self-assertively received.[57]

For analyzing the effect of packing on the execution of the proposed coordinating show we repeat MINEN the use of every GMM and K-infers. Figuredepicts this relationship Some exam portrays orchestrate lifetime due to the reality the time time period starting from the earliest place to begin of the framework execution to the use of first contraption within the framework. As can be visible from decide this regard is sort of a comparative at the same time as each GMM and K-shows are utilized in MINEN.[58] However the quantity of correspondence alters after which $30 \%$ of the facilities in the framework have left out the mark on essentialness is better for GMM than for Kmeans.

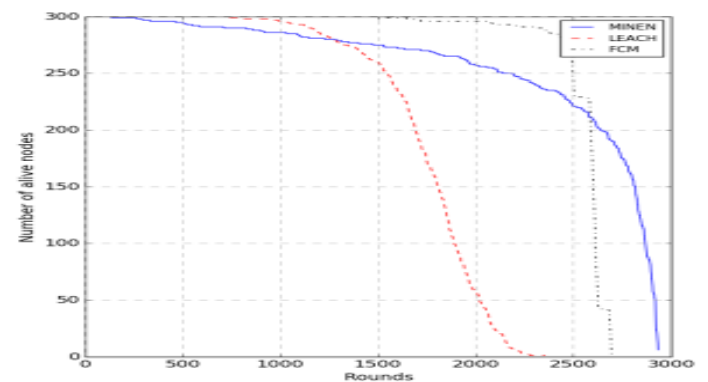

As such GMM plays well. The time out of the propagation outcomes had be situated dealt with the use of GMM in place of the bundling figuring in MINEN. Figure 4 subversions the quantity of thriving middle factors in the IoT prepare towards the quantity of slices of correspondence (stint) within the mockup.[59] As may be realized on or after the chart, in an association of 300 gadgets, MINEN can keep a hundred and fifty of those gadgets operational for as much as 2800 rounds.

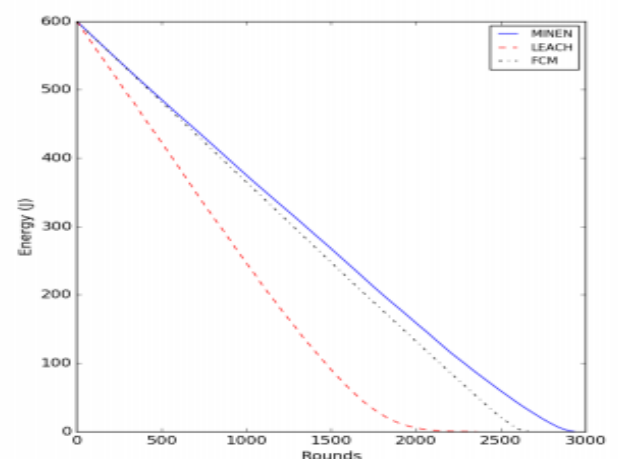

Channel and FCM of path preserve one hundred fifty gadgets dynamic certainly as much as 1700 and 2600 alters independently. Applying MINEN grows organize software's effective time to a more diploma than LEACH and FCM [60] This shows MINEN acquaint with dynamically worthwhile motion of controlling attempt amongst instruments. Figure depicts the essentialness components of the IoT kind out. Essentialness of the framework will drop right down to while all instruments miss the mark on freestyle. For MINEN this occurs after round 3000 rounds of motion, LEACH maintains the framework operational for more or less 2100 discs and FCM achieves the equal in around 2600 rounds. The grade of the outlines in like way portray that LEACH has the steepest essentialness usage charge prominent via FCM and MINEN independently. [61] Figure exhibits the amount of rounds and then $30 \%$ of the framework's devices leave out the mark on essentialness and come to be unworkable. For MINEN 30\% lessening takes place later round 2608 rounds.

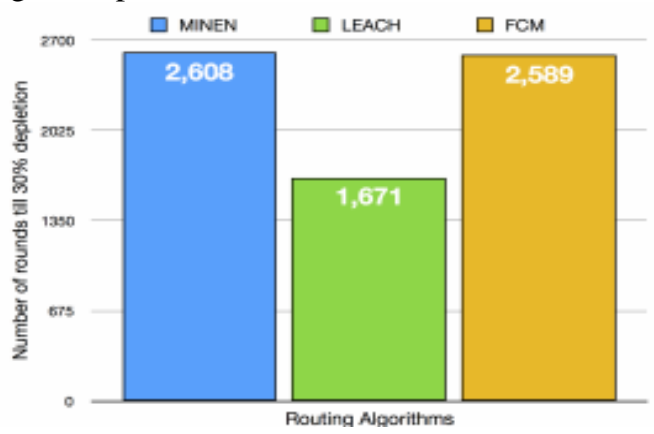

For LEACH in addition FCM these traits stand 1671 and 2589 independently. Records certainly show off the better execution of MINEN over LEACH and FCM with regard to essentialness performance. This execution is a result of a ordinary preference of capabilities utilized in collecting, recovering gathering pâte warranty measures and advent of essentialness load converting across over unalike correspondence pals in MINEN. These were comprehensively discussed within the beyond fragments. The accompanying course of movement of figures don't forget the three counts depending on framework insurance.[62] Figure highlight this examination. A purple move within the midst of those outlines represents the sordid post of the framework. The records describe the multiplication district of the IoT type out besides stand shaded in unique tones dependent proceeding to what degree gadgets in each territory are dynamic and operational. A profitable green controlling remember should most possibly keep dynamic gadgets reliably surpassed on over the framework region.[63]

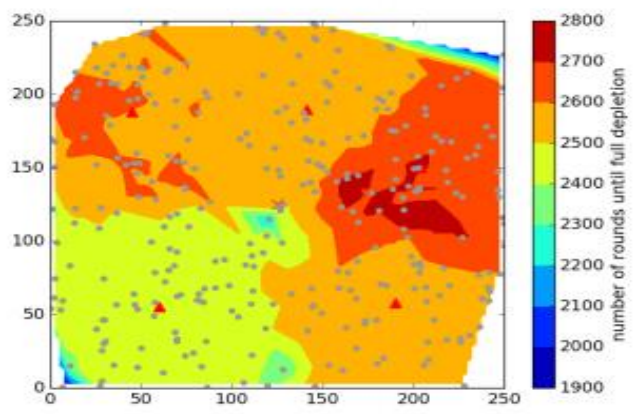

Figure 5 FCM network coverage

What's greater the operational time of contraptions need to be long. Figures five and 6 define the framework incorporation given by FCM and LEACH independently. The framework's territory location is isolated into three act areas in figure The sub district having the greatest locale (peach) on this symbol identifies with 2500 to 2600 discs up until complete weariness. The minor righthand (green) sub vicinity appears at to an effective stint of 2400-2500 adjusts but the higher right (pink) sub place identifies with 2600-2700 rounds. Consequently FCM gives uneven framework attention over the reenactment locale. [64] Figure eight reveals an impressively more non-uniform waft of framework incorporation given by means of LEACH. The quantity of slices till broad utilization of instruments in LEACH lies in the volume of a thousand to 2200 . 


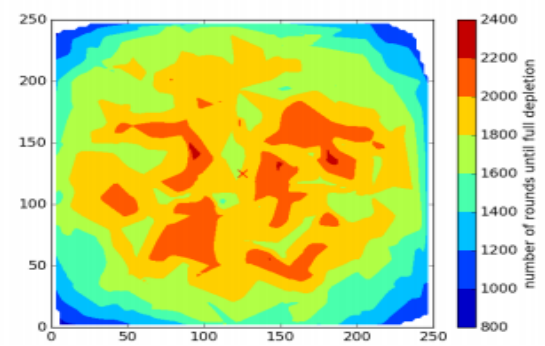

Figure 6 LEACH network coverage

The sub areas in which instruments are dynamic for longest time allotment are nearly nothing and sprinkled within the midst of the entertainment locale. As container be realized from the parent diplomacies in between beginning within supply a comparable framework attention. [65] so to talk, units shape relatively concentric circuitous sub areas, where the sub area with more variety prop up for lesser quantity of rounds. As cleared up beforehand, this occurs in mild of the fact that institution pâtés which be situated by the side of a progressively removed partition from the improper position utilize supplementary imperativeness in bestowing communications to the sordid post in LEACH. Character illustrates the framework incorporation given by means of MINEN with out rest reserving. In place of can be perceived from the determine the nice reenactment zone is recognizably blanketed inside the shade of red. This suggests large piece of gadgets within the framework are dynamic for 2400 to 3200 rings of communique.[66]

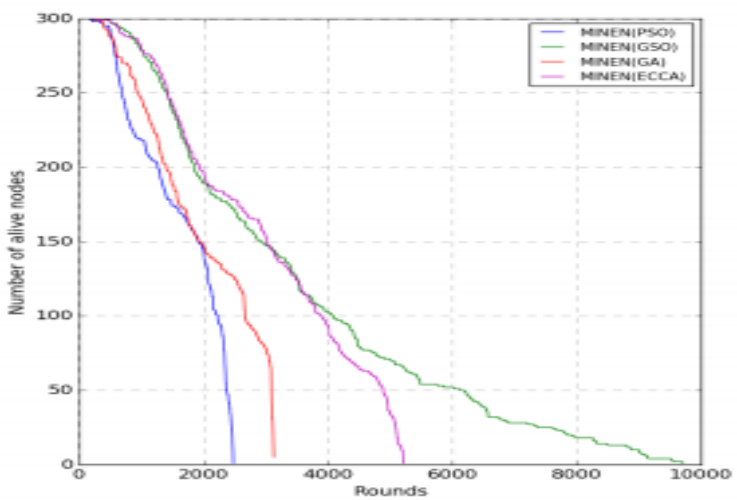

These contraptions are moreover extra reliably exceeded on over the framework's geology whilst appeared differently with regards to LEACH and FCM. Therefore MINEN gives greatest framework incorporation contemplated over in cooperation LEACH and FCM. Other than Digit 10 outlines the framework consideration of MINEN with GSO relaxation arranging It have to be located stated from this accept the execution of the framework is advanced a pair overlays by means of the use of rest development.[67] The observable blue sun sunspecs of the incorporation chart perceive with 1500-4500 rounds until complete usage of gadgets. In this way the framework remains dynamic and operational for basically greater longer time intervals than essentially using MINEN alone. To compare the feasibility of the use of GSO with MINEN we play out a close examination of various different relaxation booking estimations united with MINEN. The rest booking figurings separated are : PSO, GA and EECA.[68]

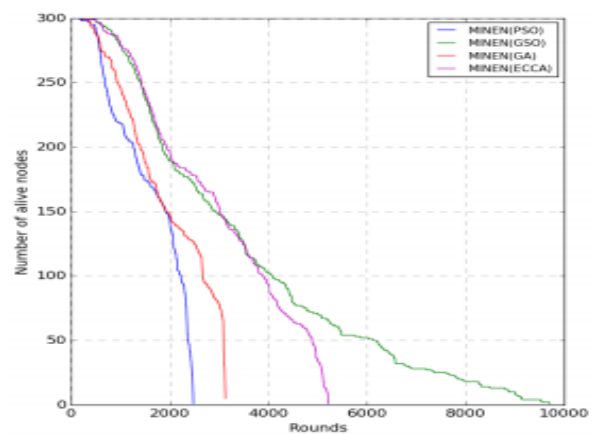

We examine these counts depending on quantity of dynamic center points and essentialness additives of the framework. Figure subversions the imperativeness of the framework with wide variety of slices of communication.[69] As can be seen from the character, MINEN with GSO engages the framework to remain operative for round 10,000 slices of correspondence. Despite the reality that MINEN with EECA, GA and PSO hold the gadgets of the framework dynamic for round 6000, 3000 and 2500 alters independently. The grades of the essentialness set against rounds (time) tables in like way aid GSO over numerous computations. PSO has the speediest charge of essentialness fatigue while GSO has negligible rate of imperativeness utilization. Figure [70] display cases the quantity of alive centers in the framework as opposed to rounds of correspondence. It rehashes the determinations introduced utilising Figure and along these strains similarly joins that MINEN while used with GSO achieves surprisingly improved than something further bleeding facet relaxation reserving computations.

\section{CONCLUSION}

One of the major basic wellsprings of WSNs is vitality protection frameworks. For wind control plant, WSNs are connected to SCADA frameworks. The remote sensors structured SCADA design in this paper will in general give bountiful constant information to wind control generators. This outfits an incredible test of unfaltering running of wind control plant. The fluffy based group plot with best picked bunch head productively diminishes the general vitality utilization esteem. Along these lines the utilization of subjective fluffy frameworks achieved multi parametric fluffy choice directing. The reenactment results demonstrate that the proposed fluffy based calculation practically expands the lifetime of the system and achieves high vitality productivity contrasted with SCADA conventions. At long last it is convinced that this class of technique will get a more noteworthy consideration and hugeness inside the exploration bunch in the up and coming years.

\section{REFERENCES}

1. I. F. Akyildiz, Weilian Su, Y. Sankarasubramaniam, and E. Cayirci. An outline on sensor frameworks. IEEE Communications Magazine, 40(8):102-114, Aug 2002.

2. J. N. Al-Karaki and A. E. Kamal.Routing strategies in remote sensor orchestrates: an examination. IEEE Wireless Communications, 11(6):6-28, Dec 2004.

3. Giuseppe Anastasi, Marco Conti, Mario Di Francesco,and Andrea Passarella. Essentialness protection in remote sensor organizes: A survey. Unrehearsed Networks, 7(3):537 - 568, 2009.

4. David Braginsky and Deborah

Estrin. Talk coordinating algorthim for sensor orchestrates In Proceedings of the first ACM 
International Workshop on Wireless Sensor Networks and Applications, WSNA '02, pages 22- 31, New York, NY, USA, 2002. ACM.

5. N. Bulusu, J. Heidemann, and D. Estrin.Gps-less insignificant exertion outside repression for little contraptions. IEEE Personal Communications, 7(5):28-34, Oct 2000.

6. AnshumanChhabra and Mariam Kiran.Grouping elephant and mice streams in quick coherent networksclassifying flows.pdf.Gotten to: 2018-05-16

7. Matthew Ettus. Structure cutoff, inertness, and power usage in multihop-guided ss-cdma remote frameworks. In Radio and Wireless Conference, 1998.RAWCON 98.1998 IEEE, pages 55- 58.IEEE, 1998.

8. JayavardhanaGubbi, RajkumarBuyya, SlavenMarusic, and MarimuthuPalaniswami. Web of things (iot): A fantasy, basic parts, and future direction. Future Generation Computer Systems, 29(7):1645 - 1660, 2013

9. Wendi RabinerHeinzelman, AnanthaChandrakasan, and HariBalakrishnan.Vitality beneficial correspondence show for remote microsensornetworks.In System sciences, 2000. Strategies of the 33rd yearly Hawaii worldwide gathering on, pages 10- pp. IEEE, 2000

10. Wendi RabinerHeinzelman, Joanna Kulik, and HariBalakrishnan.Versatile shows for information dispersal in remote sensor frameworks. In Proceedings of the fifth Annual ACM/IEEE International Conference on Mobile Computing and Networking, MobiCom '99, pages 174-185, New York, NY, USA, 1999. ACM.

11. DC Hoang, R Kumar, and SK Panda. Fleecy c-suggests grouping show for remote sensor frameworks. In Industrial Electronics (ISIE), 2010 IEEE International Symposium on, pages 3477- 3482.IEEE, 2010.

12. Jun Huang, Yu Meng, Xuehong Gong, Yanbing Liu, and QiangDuan. A story association scheme for green trap of things. IEEE Internet of Things Journal, 1(2):196-205, 2014.

13. JieJia, Jian Chen, Guiran Chang, and Zhenhua Tan.Vitality capable consideration control in remote sensor frameworks reliant on multi-objective innate estimation. PCs and Mathematics with Applications, 57(11):1756 - 1766, 2009. Strategies of the International Conference.

14. Sumit Kumar and Siddhartha Chauhan.A survey on booking estimations for remote sensor systems.Global Journal of Computer Applications, 20(5), 2011.

15. Andreas Savvides, Chih-Chieh Han, and Mani B. Strivastava.Dynamic fine-grained limitation in extraordinarily delegated frameworks of sensors. In Proceedings of the seventh Annual International Conference on Mobile Computing and Networking, MobiCom '01, pages 166-179, New York, NY, USA, 2001. ACM.

16. Curt Schurgers and Mani B Srivastava.Vitality successful directing in remote sensor frameworks. In Military exchanges meeting, 2001 MILCOM 2001.Interchanges for framework driven exercises: Creating the information compel.IEEE, volume 1, pages 357-361.IEEE, 2001.

17. Faisal KarimShaikh, SheraliZeadally, and Ernesto Exposito.Empowering progressions for green snare of things. IEEE Systems Journal, 11(2):983-994, 2017

18. K. Sohrabi, J. Gao, V. Ailawadhi, and G. J. Pottie. Shows for selforganization of a remote sensor mastermind. IEEE Personal Communications, 7(5):16-27, Oct 2000.

19. L. Subramanian and R. H. Katz.An building for structure selfconfigurable systems. In 2000 First Annual Workshop on Mobile and Ad Hoc Networking and Computing. MobiHOC (Cat. No.00EX444), pages 63- 73, 2000

20. Fan Ye, A. Chen, Songwu Lu, and Lixia Zhang. An adaptable response for least cost sending in immense sensor frameworks. In Proceedings Tenth International Conference on Computer Communications and Networks (Cat. No.01EX495), pages 304- 309, 2001

21. Chunsheng Zhu, Victor CM Leung, Lei Shu, and Edith C-H Ngai.Green web of things for quick world. IEEE Access, 3:2151-2162, 2015.

22. M Bahrololum and M Khaleghi. Irregularity interference disclosure system using gaussian mix illustrate. In Convergence and Hybrid Information Technology, 2008.ICCIT'08. Third International Conference on, volume 1,pages 1162-1167. IEEE, 2008.

23. Xiaohong Sheng, $\mathrm{Y}-\mathrm{H} \mathrm{Hu}$, and ParameswaranRamanathan Appropriated molecule channel with gmm infer for different targets constrainment and following in remote sensor sort out. In Information Processing in Sensor Networks, 2005.IPSN 2005. Fourth International Symposium on, pages 181- 188. IEEE, 2005.

24. ChhabraAnshuman, VashishthVidushi, and Sharma Deepak Kumar A feathery reason and delight speculation based adaptable procedure for confirming spearheading frameworks against dull hole attacks.

Worldwide Journal of Communication Systems, 31(4):e3487. e3487 IJCS-17-0453.R1.

25. A. Chhabra, V. Vashishth, and D. K. Sharma. A delight theory based secure model against dim hole attacks in quick frameworks. In 2017 51st Annual Conference on Information Sciences and Systems (CISS), pages 1-6, March 2017.

26. R. Di Pietro, S. Guarino, N.V. Verde, and J. Domingo-Ferrer.Security in remote uniquely designated frameworks 2013 a review. Computer Communications, 51:1 - 20, 2014.

27. A. Chhabra, V. Vashishth, and D. K. Sharma.Seir: A stackelberg redirection based technique for imperativeness careful and helped coordinating in extremist spearheading frameworks. In $201751 \mathrm{~s}$ Annual Conference on Information Sciences and Systems (CISS), pages 1-6, March 2017.

28. C. Yu, W. Guo, and G. Chen.Vitality balanced lay arranging subject to atom swarm streamlining in remote sensor organize. In 2012 IEEE 26th International Parallel and Distributed Processing Symposium Workshops PhD Forum, pages 1249- 1255, May 2012.

29. J. Kennedy, "Atom swarm improvement," in Encyclopedia of AI Springer, 2011, pp. 760- 766.

30. [30] I. F. Akyildiz , Weilian Su , Y. Sankarasubramaniam , E. Cayirci, An outline on sensor frameworks, IEEE Communications Magazine, v.40 n.8, p.102-114,

31. M. Li, C. Hua, C. Chen, X. Guan, "Application-driven virtual network embedding for industrial wireless sensor networks", 2017 IEEE International Conference on Communications (ICC) Ad-Hoc and Sensor Networking Sumposium, 21-25 May 2017.

32. R. Rajagopalan , P. K. Varshney, Data-total systems in sensor organizes: an overview, IEEE Communications Surveys and Tutorials, v. 8 n. 4, p. $48-63$

33. TossapornSrisooksai, KamolKeamarungsi, PoonlapLamsrichan Kiyomichi Araki, Practical information pressure in remote sensor arranges: A study, Journal of Network and Computer Applications, v.35 n.1, p.37-59,

34. V. Katiyar, N. Chand, S. Soni, "Grouping calculations for heterogeneous remote sensor arrange: a study", International Journal of Applied Engineering Research, Dindigul, Volume 1, No 2, 2010, pp. 273- - 287.

35. F. Alfayez, M. Hammoudeh, A. Abuarqoub, "A Survey on MAC conventions for obligation cycled remote sensor systems", International Conference on Advanced Wireless Information and Communication Technologies (AWICT 2015), Science Direct, Procedia Computer Science, Volume 73. 2015, PP: 482- - 489.

36. J. N. Al-Karaki , A. E. Kamal, Routing procedures in remote sensor organizes: a study, IEEE Wireless Communications, v.11 n.6, p.6-28,

37. N. A. Pantazis, S. A. Nikolidakis, D. D. Vergados, "Vitality productive steering conventions in remote sensor organizes: a review", IEEE Communications Surveys and Tutorials (Volume: 15, Issue: 2), pp 551- - 591. 2013.

38. Wajgi, N. V. Thakur., "Burden adjusting calculations in remote sensor organize: a review", IRACST - International Journal of Computer Networks and Wireless Communications ISSN: 2250- - 3501. Vol.2 No 4, August 2012, pp. 456- - 460

39. Faisal B. Hussain, YalcinCebi , Ghalib A. Shah, A multievent blockage control convention for remote sensor systems, EURASIP Journal on Wireless Communications and Networking,

40. G. A. Shah, M. Bozyiğit, Ö. B. Akan, "Multi-occasion versatile bunching (MEAC) convention for heterogeneous remote sensor systems", Proc. The MP Fifth Annual Mediterranean Ad Hoc Networking Workshop (Med-Hoc-Net 2006), pp. 30- - 37

41. A. V. Sutagundar, S. S.Manvi, "Area mindful occasion driven multipath steering in Wireless Sensor Networks: Agent based methodology". Egyptian Informatics Journal, Volume 14, Issue 1 , March 2013, pp. 55- - 65

42. C. F. García-Hernández, P. H. Ibargüengoytia-González, J. García-Hernández, J. A. Pérez-Díaz, "Remote sensor systems and applications: an overview". IJCSNS International Journal of Computer Science and Network Security, Vol.7 No.3, March 2007, pp. 264273

43. MauriKuorilehto, Marko Hännikäinen, Timo D. Hämäläinen, A study of use dispersion in remote sensor systems, EURASIP Journal on Wireless C[39] Faisal B. Hussain, YalcinCebi , Ghalib A. Shah, A multievent clog control convention for remote sensor systems, EURASIP Journal on Wireless Communications and Networking, 
44. G. A. Shah, M. Bozyiğit, Ö. B. Akan, "Multi-occasion versatile bunching (MEAC) convention for heterogeneous remote sensor systems", Proc. The MP Fifth Annual Mediterranean Ad Hoc Networking Workshop (Med-Hoc-Net 2006), pp. 30- - 37

45. A. V. Sutagundar, S. S.Manvi, "Area mindful occasion driven multipath directing in Wireless Sensor Networks: Agent based methodology". Egyptian Informatics Journal, Volume 14, Issue 1, March 2013, pp. 55- - 65

46. C. F. García-Hernández, P. H. Ibargüengoytia-González, J. García-Hernández, J. A. Pérez-Díaz, "Remote sensor systems and applications: a review". IJCSNS International Journal of Computer Science and Network Security, Vol.7 No.3, March 2007, pp. 264- 273

47. MauriKuorilehto, Marko Hännikäinen, Timo D. Hämäläinen, A study of use dissemination in remote sensor systems, EURASIP Journal on Wireless Communications and Networking, v.2005 n.5, p.774-788, October 2005

48. Dongjin Son , BhaskarKrishnamachari , John Heidemann, Experimental investigation of simultaneous transmission in remote sensor systems, Proceedings of the fourth universal gathering on Embedded organized sensor frameworks, October 31-November 03 , 2006

49. J. Rang, Y. Zhang, B. Nath, "Start to finish Channel Capacity Measurement for Congestion Control in Sensor Networks", In Proceedings of the second International Workshop on Sensor an Actor Network Protocols and Applications (SANPA '04), Boston, MA, USA, 22 August 2004. communications and Networking, v.2005 n.5, p.774-788, October 2005

50. Dongjin Son , BhaskarKrishnamachari , John Heidemann, Experimental investigation of simultaneous transmission in remote sensor systems, Proceedings of the fourth worldwide gathering on Embedded organized sensor frameworks, October 31-November 03 2006

51. J. Rang, Y. Zhang, B. Nath, "From beginning to end Channel Capacity Measurement for Congestion Control in Sensor Networks", In Proceedings of the second International Workshop on Sensor an Actor Network Protocols and Applications (SANPA '04), Boston, MA, USA, 22 August 2004.

52. M. Radi, B. Dezfouli, K. A. Bakar, M. Lee, "Multipath steering in remote sensor organizes: a review and research difficulties", Sensors ISSN 1424-8220, 2012, 12, pp. 650- - 685

53. M. Masdari, M. Tanabi., "Multipath directing conventions in remote sensor arranges: a review and investigation", International Journal of Future Generation Communication and Networking, Vol.6, No.6, 2013 , pp. 181- - 192

54. Budhaditya Deb, SudeeptBhatnagar, BadriNath, ReInForM: Reliable Information Forwarding Using Multiple Paths in Sensor Networks, Proceedings of the 28th Annual IEEE International Conference on Local Computer Networks, p.406, October 20-24, 2003

55. M. Cherian, T. R. G. Nair "Multipath Routing with Novel Packet Scheduling Approach in Wireless Sensor Networks", International Journal of Computer Theory and Engineering 3(5), pp. 666- - 670, 2011

56. T. Cinar and M. BulentOrencik, "A submerged acoustic channel demonstrate utilizing beam following in ns-2," in Proceedings of the 2009 second IFIP Wireless Days (WD 2009), pp. 1-6

57. M. Ayaz and A. Abdullah, "Submerged remote sensor systems Routing issues and future difficulties," in Proceedings of seventh International Conference on Advances in Mobile Computing and Multimedia, pp. 370-375

58. L. Liu, S. Zhou, and J. H. Cui, "Prospects and problems of wireless communication for underwater sensor networks," Wireless Communications and Mobile Computing, vol. 8, no. 8, pp. 977-994, 2008.

59. G. Zaibi, N. Nasri, A. Kacouri, and M. Samet, "Survey of temperature variation effect on underwater acoustic wireless transmission,” ICGST-CNIR Journal, vol. 9, 2009.

60. Y. Luo, L. Pu, M. Zuba, "Challenges and opportunities of underwater cognitive acoustic networks," IEEE Transactions on Emerging Topics in Computing, vol. 2, no. 2, pp. 198-211, 2014.

61. X.- P. Gu, Y. Yang, and R.- L. Hu, "Breaking down the execution of station in Underwater Wireless Sensor Networks(UWSN)," in Proceedings of the 2011 International Conference on Advanced in Control Engineering and Information Science, CEIS 2011, pp. 95-99, China, August 2011.

62. J. Kong, M. Gerla, and S. Zhou, Challenges: Building Scalable and Distributed Underwater Wireless Sensor Networks (UWSNs) for Aquatic Applications, 2005.
63. S. Basagni, C. Petrioli, R. Petroccia, and M. Stojanovic, "Improved bundle measure determination in submerged remote sensor organize correspondences," IEEE Journal of Oceanic Engineering, vol. 37, no. 3, pp. 1-337, 2012.

64. S. Basagni, C. Petrioli, R. Petroccia, and M. Stojanovic, "Streamlining system execution through bundle discontinuity in multi-bounce submerged correspondences," in Proceedings of the OCEANS'10 IEEE Sydney, OCEANSSYD 2010, pp. 1- 7, Australia, May 2010.

65. S. Basagni, C. Petrioli, R. Petroccia, and M. Stojanovic, "Picking the parcel estimate in multi-bounce submerged systems," in Proceedings of the OCEANS'10 IEEE Sydney, OCEANSSYD 2010, pp. 1-9, Australia, May 2010

66. M. Ayaz, L. T. Jung, A. Abdullah, and I. Ahmad, "Solid information conveyances utilizing parcel advancement in multi-jump submerged sensor systems," Journal of King Saud University - Computer and Information Sciences, vol. 24, no. 1, pp. 41-48, 2012.

67. N. Li, J.-F. Martínez, J. M. M. Chaus, and M. Eckert, "A survey on underwater acoustic sensor network routing protocols," Sensors, vol 16, no. 3, p. 414, 2016.

68. M. Faheem, G. Fish, and V. C. Gungor, "QERP: Quality-of-Service (QoS) Aware Evolutionary Routing Protocol for Underwater Wireless Sensor Networks," IEEE Systems Journal, 2017.

69. C. Zidi, F. Bouabdallah, and R. Boutaba, "Directing structure dodging vitality openings in submerged acoustic sensor systems," Wireless Communications and Mobile Computing, vol. 16, no. 14, pp. 2035 2051, 2016

70. N. Z. Zenia, M. Aseeri, M. R. Ahmed, Z. I. Chowdhury, and M Shamim Kaiser, "Vitality effectiveness and dependability in MAC and directing conventions for submerged remote sensor organize: A review," Journal of Network and Computer Applications, vol. 71, pp. $72-85,2016$ 